\title{
Neutrino Magnetic Moments, Flavor Mixing, and the Super-Kamiokande Solar Data
}

\author{
J.F. Beacom and P. Vogel \\ Physics Department 161-33, California Institute of Technology, Pasadena, California 91125
}

(Received 16 July 1999)

\begin{abstract}
We find that magnetic neutrino-electron scattering is unaffected by oscillations for vacuum mixing of Dirac neutrinos with only diagonal moments and for Majorana neutrinos with two flavors. For Mikheyev-Smirnov-Wolfenstein mixing, these cases are again obtained, though the effective moments can depend on the neutrino energy. Thus, e.g., the magnetic moments measured with $\bar{\nu}_{e}$ from a reactor and $\nu_{e}$ from the Sun could be different. With minimal assumptions, we find a new limit on $\mu_{\nu}$ using the 825-d Super-Kamiokande solar neutrino data: $\left|\mu_{\nu}\right| \leq 1.5 \times 10^{-10} \mu_{B}$ at $90 \% \mathrm{CL}$, comparable to the existing reactor limit.
\end{abstract}

PACS numbers: 14.60.Pq, 13.15.+g, 13.40.Em, 26.65. $+\mathrm{t}$

In the minimally extended standard model, neutrinos of mass $m_{\nu}$ have tiny loop-induced magnetic moments $\mu_{\nu} \simeq 3 \times 10^{-19} \mu_{B}\left[m_{\nu} /(1 \mathrm{eV})\right]$, where $\mu_{B}$ is the Bohr magneton. In various extensions of the standard model, larger magnetic moments can occur without large neutrino masses. In the presence of flavor mixing, the fundamental magnetic moments are associated with the mass eigenstates (since either a boost or a magnetic moment can be used to reverse the helicity). In the mass eigenstate basis, Dirac neutrinos can have diagonal or off-diagonal (transition) moments, while Majorana neutrinos can have only transition moments $[1,2]$.

In the current experiments, the effects of neutrino magnetic moments can be searched for only in the recoil electron spectrum from neutrino-electron scattering $[3,4]$. Below we consider the interplay between magnetic moments and flavor mixing for this process. We show how magnetic moments can be defined for beams that are initially neutrino flavor eigenstates. In some important cases these moments do not oscillate, i.e., they do not depend on distance from the source. However, in the presence of Mikheyev-Smirnov-Wolfenstein (MSW) mixing, these defined flavor moments can differ from the vacuum case and can depend on the neutrino energy, though not on the distance. As an illustration, we derive a new limit on the magnetic moment from the SuperKamiokande (SK) solar neutrino data [5].

There are two incoherent contributions to neutrinoelectron scattering: weak scattering, which preserves the neutrino helicity, and magnetic scattering, which reverses it. Thus the differential cross section is given by

$$
\begin{aligned}
\frac{d \sigma}{d T}= & \frac{2 G_{F}^{2} m_{e}}{\pi}\left[g_{L}^{2}+g_{R}^{2}\left(1-\frac{T}{E_{\nu}}\right)^{2}-g_{L} g_{R} \frac{m_{e} T}{E_{\nu}^{2}}\right] \\
& +\mu_{\nu}^{2} \frac{\pi \alpha^{2}}{m_{e}^{2}} \frac{1-T / E_{\nu}}{T} .
\end{aligned}
$$

In Eq. (1), $g_{L}=\sin ^{2} \theta_{W}+1 / 2$ for $\nu_{e}, g_{L}=\sin ^{2} \theta_{W}-$ $1 / 2$ for $\nu_{\mu}$ and $\nu_{\tau}$, and $g_{R}=\sin ^{2} \theta_{W}$ for all flavors (for antineutrinos, exchange $g_{L}$ and $g_{R}$ ). The magnetic moment $\mu_{\nu}$ is expressed in units of $\mu_{B}$. Magnetic scattering, the second term in Eq. (1), grows rapidly with decreasing electron recoil kinetic energy $T$.

In principle, there can be weak-magnetic interference effects. There is a negligible effect due to the fact that a massive neutrino is not a helicity eigenstate [6]. Also, if the neutrinos have a transverse polarization, the electron azimuthal angle distribution can be affected [7]; we ignore this case, as the effects are presently unobservable.

Vacuum mixing. - The effects of flavor mixing on the weak scattering are well known. Whatever the composition of the neutrino beam, the different flavors are in principle distinguishable and hence their cross sections combine incoherently, weighted by the probabilities for the neutrino to be of each given flavor.

We want to explore how neutrino oscillations affect the magnetic scattering. The shape of the electron recoil spectrum in magnetic scattering is universal (the same for all mass eigenstates). The only quantity that depends on the beam composition is the effective magnetic moment $\mu_{\nu}$. Let us assume that we begin with a beam of electron neutrinos. Under the usual oscillation hypothesis such a beam propagates over the distance $L$ from its source in vacuum according to

$$
\left|\nu_{e}(L)\right\rangle=\sum_{k} U_{e k} e^{-i E_{k} L}\left|\nu_{k}\right\rangle,
$$

where $U_{e k}$ is an element of the unitary mixing matrix and $k$ labels the mass eigenstates.

Similarly to above, whatever the composition of the neutrino beam, the different mass eigenstates are in principle distinguishable in the magnetic scattering, and hence their cross sections combine incoherently, weighted by the squares of the amplitudes for the neutrino to be of each mass after the scattering. Then the combined cross section for magnetic scattering has the form of Eq. (1) with magnetic moment squared $\mu_{\nu}^{2}$ given by

$$
\begin{aligned}
\mu_{e}^{2} & =\sum_{j}\left|\sum_{k} U_{e k} e^{-i E_{k} L} \mu_{j k}\right|^{2} \\
& =\sum_{j} \sum_{k k^{\prime}} U_{e k} U_{e k^{\prime}}^{*} \mu_{j k} \mu_{j k^{\prime}}^{*} e^{-2 \pi i L / L_{k k^{\prime}}},
\end{aligned}
$$


where the summations $j, k, k^{\prime}$ are over the mass eigenstates and the subscript $e$ labels the initial flavor. We have made the usual relativistic expansion and have defined the oscillation length $L_{k k^{\prime}}=4 \pi E_{\nu} / \Delta m_{k k^{\prime}}^{2}$ for $k \neq k^{\prime}$ (there is no $L$ dependent phase for $k=k^{\prime}$ ). The quantities $\mu_{j k}$ in Eq. (3) are the fundamental constants (in units of $\mu_{B}$ ) that characterize the coupling of the neutrino mass eigenstates to the electromagnetic field. The summation over $j$ is outside the square because of the incoherence of the cross sections for different final masses. The expression for $\mu_{e}^{2}$ simplifies in some important cases.

Let us assume first that the neutrinos are Dirac particles (with $n$ flavors) with only diagonal magnetic moments $\left(\mu_{j k}=\mu_{j} \delta_{j k}\right)$; this is the scenario used by the Particle Data Group [8]. Then

$$
\mu_{e}^{2}=\sum_{j}\left|U_{e j}\right|^{2}\left|\mu_{j}\right|^{2},
$$

and there is no dependence on the distance $L$ or neutrino energy $E_{\nu}$. In this case one can characterize the magnetic scattering by the initial flavor index instead of the mass indices. (Hence we disagree with Ref. [9], in which the magnetic scattering depends on the final flavor index; i.e., it oscillates.) Measurements of all magnetic moments and mixing parameters would allow extraction of the "fundamental" moments $\mu_{j}$.

Next consider the case of Majorana neutrinos and assume that only two mass eigenstates are relevant. Then

$$
\mu_{e}^{2}=\left|\mu_{12}\right|^{2}\left(\left|U_{e 1}\right|^{2}+\left|U_{e 2}\right|^{2}\right)=\left|\mu_{12}\right|^{2},
$$

which is not only independent of the source distance and the neutrino energy, but also of the mixing angle.

Under what circumstances does one have to worry about a dependence on distance and neutrino energy, and, in particular, when can such terms be dominant? Clearly, at least one term of the type $\mu_{j k} \times \mu_{j k^{\prime}}$ with $k \neq k^{\prime}$ must be nonvanishing and as large as $\mu_{j k}^{2}$ or $\mu_{j k^{\prime}}^{2}$. In other words, in the $3 \times 3$ matrix $\mu_{j k}$ there should be at least two comparable entries on the same line (and in the same column). For the Dirac case this implies that at least one nondiagonal magnetic moment is as large as the diagonal ones. For the Majorana case it implies that $t w o$ different nondiagonal magnetic moments are of a similar magnitude. Both of these cases seem unnatural.

MSW mixing. - The above discussion must be modified for matter-enhanced oscillations (the MSW effect). First, the initial composition of the beam is governed not by the vacuum mixing angle $\theta_{v}$, but by the initial matter mixing angle $\theta_{m}$, which depends on $\Delta m^{2} / 2 E_{\nu}$ and the electron density. If the initial density is well above the resonance density, as is true for the standard solutions to the solar neutrino problem, then $\theta_{m} \simeq \pi / 2$ to an excellent approximation. Then initially, $\left|\nu_{e}\right\rangle=$ $\cos \theta_{m}\left|\nu_{1}\right\rangle+\sin \theta_{m}\left|\nu_{2}\right\rangle \simeq\left|\nu_{2}\right\rangle$.
Second, although a nearly pure $\left|\nu_{2}\right\rangle$ is produced in the solar center, if the passage through the resonance is nonadiabatic, then the final beam can be a mixture of $\left|\nu_{1}\right\rangle$ and $\left|\nu_{2}\right\rangle$. Most generally [10], the mass eigenstates evolve as

$$
\begin{aligned}
& \left|\nu_{1}\right\rangle \rightarrow c_{1} e^{+i \phi_{a}}\left|\nu_{1}\right\rangle+c_{2} e^{+i \phi_{b}}\left|\nu_{2}\right\rangle, \\
& \left|\nu_{2}\right\rangle \rightarrow-c_{2}^{*} e^{-i \phi_{b}}\left|\nu_{1}\right\rangle+c_{1}^{*} e^{-i \phi_{a}}\left|\nu_{2}\right\rangle,
\end{aligned}
$$

where $\left|c_{1}\right|^{2}+\left|c_{2}\right|^{2}=1$. The phases $\phi_{a}$ and $\phi_{b}$ (real functions that depend on integrals of the instantaneous mass basis eigenvalues) are irrelevant here, due to the noninterference of different mass eigenstates in the magnetic scattering. For the adiabatic case (e.g., the solar largeangle solution [11]), $c_{2}=0$. For the nonadiabatic case (e.g., the solar small-angle solution [11]), and a narrow resonance region (which naturally obtains), the probability of hopping from one mass eigenstate to the other is $P_{\text {hop }}=$ $\left|c_{2}\right|^{2}$, which depends on the neutrino energy but not the distance from the source, e.g., for an exponential density with density scale height $r_{s}$,

$$
P_{\text {hop }}=\exp \left[-\pi \frac{\Delta m^{2}}{2 E_{\nu}} r_{s}\left(1-\cos 2 \theta_{v}\right)\right] .
$$

Thus for two-flavor Dirac mixing with only diagonal moments, we obtain for the effective magnetic moment

$$
\begin{aligned}
\mu_{e}^{2} & =\left|c_{2}\right|^{2}\left|\mu_{1}\right|^{2}+\left|c_{1}\right|^{2}\left|\mu_{2}\right|^{2} \\
& =P_{\text {hop }}\left|\mu_{1}\right|^{2}+\left(1-P_{\text {hop }}\right)\left|\mu_{2}\right|^{2} .
\end{aligned}
$$

Note that this is different from Eq. (4), even in the adiabatic case. However, for the two-flavor Majorana case, we again obtain $\mu_{e}^{2}=\left|\mu_{12}\right|^{2}$, as in Eq. (5). In both cases, since the initial state is a pure $\left|\nu_{2}\right\rangle$, there are no interference terms that depend on distance.

SK data. - The best direct limit on the neutrino magnetic moment, $1.8 \times 10^{-10} \mu_{B}$ at $90 \%$ C.L. [12], comes from studies of neutrino-electron scattering with reactor antineutrinos. (See Ref. [13] and references therein for the astrophysical limits.) As explained above, the meaning of the measured $\mu_{\nu}$ using solar neutrinos and reactor antineutrinos could in principle be different. Nevertheless, it is important to realize that a magnetic moment numerically equal to the current reactor limit would have a statistically significant effect on the solar neutrino data from SK. Since there is, as explained below, no evidence in the data for a nonvanishing magnetic moment, we derive, with a minimum of assumptions, a limit on what we call $\mu_{e}^{\text {sol }}$.

If the expected weak scattering rate were known (as assumed in Ref. [14]), an observed excess in the total rate would indicate a nonzero magnetic moment. However, as the total weak rate is not known a priori, we instead look at the shape of the electron spectrum for the effects 
of a magnetic moment. The signature of a nonvanishing magnetic moment would be an enhancement, compared to the weak scattering alone, of the events at low recoil energies, with less enhancement at higher energies. That is not observed. Instead, as shown below, the electron spectra recorded by SK have, within the statistics, the shape that one expects from weak scattering alone (we show below that the deviations observed currently in the highest energy bins are irrelevant for our purpose). However, the total number of events is less than the standard solar model predicts, presumably due to neutrino oscillations. We do not need to know the value, or the mixing mechanism behind it, of this overall reduction of the scattering rate.

We assume only that the shape of the measured spectrum is not due to a fortuitous cancellation between a magnetic moment effect rising at low energies and an oscillation effect rising at high energies. The Sudbury Neutrino Observatory will check the spectral shape and total flux of the $\nu_{e}$ component.

The procedure we adopt uses the measured relative errors by SK and the fact that the measured shape agrees with expectations. We calculate $\langle d \sigma / d T\rangle$ by folding Eq. (1) with the ${ }^{8} B$ neutrino spectrum from Ref. [15]. For both weak and magnetic scattering, we include the SK energy resolution [16], though it makes little difference in the final results. We histogram the results in $0.5 \mathrm{MeV}$ bins in total electron energy, as in SK. Thus, as a function of the bin number $i$, we have constructed the expected spectra $n_{W}(i)$ and $n_{M}(i)$ for weak and magnetic scattering, respectively.

In order to determine the upper limit of $\left|\mu_{e}^{\text {sol }}\right|^{2}$ we must take into account the statistical fluctuations in the SK data. While the data points divided by the solar model expectation are consistent with an energy independent reduction factor $\alpha$, the individual bins are distributed, presumably randomly, around that value. To take that into account we choose some reference values $\alpha_{\text {ref }}$ and $\mu_{\text {ref }}$ and create a set of simulated data, $n_{S}(i)$, which are Gaussian distributed around the theoretical expectation $\alpha_{\text {ref }} n_{W}(i)+\mu_{\text {ref }}^{2} n_{M}(i)$ with the relative errors $\sigma(i)$ given by SK.

We then minimize the $\chi^{2}$,

$$
\chi^{2}=\sum_{i}\left[\frac{\alpha n_{W}(i)+\mu_{\nu}^{2} n_{M}(i)-n_{S}(i)}{\sigma(i) n_{S}(i)}\right]^{2},
$$

with respect to the fit parameters $\alpha$ and $\mu_{\nu}^{2}$. For fixed $\alpha_{\text {ref }}$ and $\mu_{\text {ref }}^{2}$, we repeat this procedure many times and plot the frequencies with which given values of the fitted $\alpha$ and $\mu_{\nu}^{2}$ appear. An example of the scatter plot of the fit parameters is shown in Fig. 1. One can see, naturally, that the most probable values of the fit are the reference values $\alpha_{\text {ref }}$ and $\mu_{\text {ref }}$. Also, the two variables are strongly anticorrelated (correlation coefficient $r \simeq-0.9$ ); i.e., larger $\alpha$ is accompanied by smaller $\mu_{\nu}^{2}$. Dividing

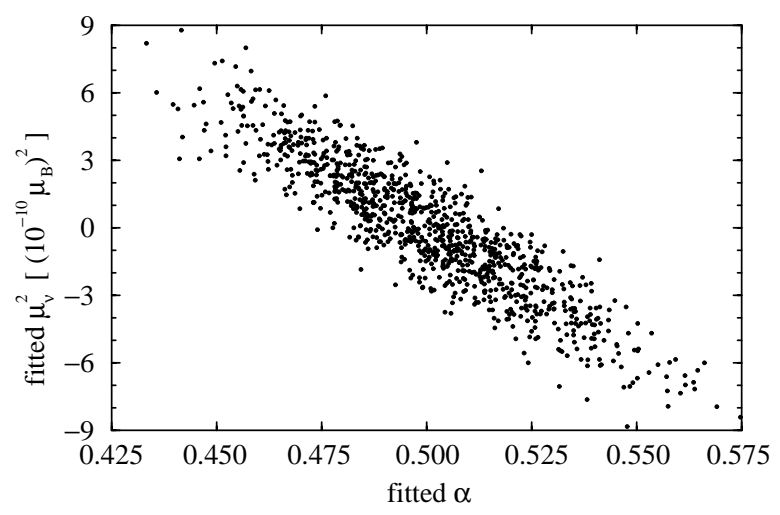

FIG. 1. Scatter plot illustrating the ranges and frequencies with which the fitted $\alpha$ and $\mu_{\nu}^{2}$ appear. The plot corresponds to $\alpha_{\text {ref }}=0.5$ and $\mu_{\text {ref }}=0$ and uses the relative errors (504-d data) of Ref. [5].

the numerator and denominator in Eq. (10) by $n_{W}(i)$, one sees that the $\chi^{2}$ depends only on $r_{i}=n_{S}(i) / n_{W}(i)$, i.e., precisely on the quantities published by SK [5].

By repeating the calculation for different values of $\mu_{\text {ref }}$ and projecting on the $\mu_{\nu}^{2}$ axis, one gets the distributions shown in the upper panel of Fig. 2. These distributions are Gaussian, and their width is almost independent of the chosen value of $\mu_{\text {ref }}$. Based on them we obtain the lines in the lower panel of Fig. 2 signifying confidence levels at $10 \%, 50 \%$ (the mean), and $90 \%$. For a given fitted $\mu_{\nu}^{2}$ obtained in an experiment, these allow one to determine the likely range of the true $\mu_{\nu}^{2}$. For example, if one found a fitted $\mu_{\nu}^{2} \simeq 4$, then from Fig. 2, the most probable true value is $\mu_{\nu}^{2} \simeq 4$, with the upper limit being $\simeq 8$ and the lower limit being $\simeq 0$. Similarly, for a fitted $\mu_{\nu}^{2} \simeq 0$, the

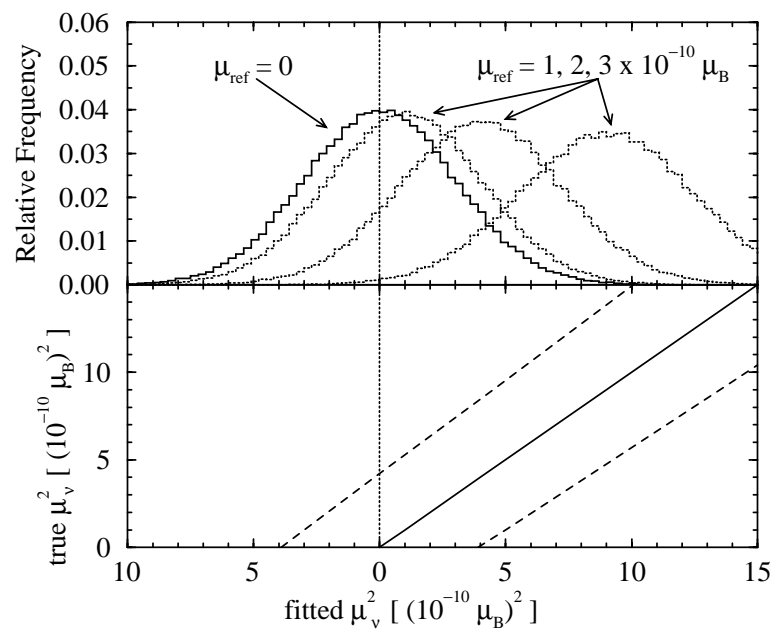

FIG. 2. The upper panel shows the relative frequencies of different fitted $\mu_{\nu}^{2}$ values for the indicated values of $\mu_{\text {ref }}$ and for $\alpha_{\text {ref }}=0.5$. The calculation uses the relative errors (504-d data) of Ref. [5]. The lower panel allows one to find for any fitted $\mu_{\nu}^{2}$ the most probable value of the true $\mu_{\nu}^{2}$ (full line) and the $10 \%$ and the $90 \%$ C.L. interval of that quantity (dashed lines). 
true $\mu_{\nu}^{2}$ is $\lesssim 3.9$. This would be the largest true $\mu_{\nu}^{2}$, due to statistical fluctuations of the finite data, that could have given this fitted $\mu_{\nu}^{2} \simeq 0$.

In this way we solve problems associated with the statistical fluctuations as well as with the constraint $\left|\mu_{e}^{\text {sol }}\right|^{2} \geq 0$. Figure 2 was calculated with $\alpha_{\text {ref }}=0.5$, as observed in SK, but does not change significantly for $0.4<\alpha_{\text {ref }}<0.6$. Note that the results summarized in Fig. 2 can be also obtained analytically, without generating many simulated spectra. The conclusions, in particular, the lower panel of Fig. 2, simply follow from the properties of the individual sums in Eq. (10).

Using the SK data [5], the fitted $\alpha \simeq 0.5$; the exact value is irrelevant since we are testing only the spectral shape, and not the normalization. The fitted values of $\mu_{\nu}^{2}$ are slightly (but not significantly; see Fig. 2) negative: $\simeq-5$, -3 , and -2 (in the units of Table I) for the 504-, 708-, and 825-day data sets. The slight (but diminishing with time) positive slope observed in the data cannot be caused by a magnetic moment (which causes an increase at low energies), though it could be caused by oscillations. The most conservative conclusion is therefore to say that the slope is not negative, i.e., that the fitted $\mu_{\nu}^{2}$ values are not positive. That is, we obtain the limit by using Fig. 2 (and its analogs) and an assumed fitted value of $\mu_{\nu}^{2}=0$. Thus the limits in Table I are slightly weaker than what is naively implied by the data, but are more robust. The sensitivity to $\left|\mu_{e}^{\text {sol }}\right|$ improves with time only as $t^{-1 / 4}$, but the addition of more low-energy bins (e.g., the two added since the 504-d data) gives a more dramatic improvement.

The uncertainties $\delta \alpha$ in Table I reflect the increase in the error in the parameter $\alpha$ when one allows magnetic scattering. Our procedure does not include the correlations between systematic errors in different bins and therefore will not reflect the full systematic uncertainty. Note that in the standard analysis [5] one assumes $\mu_{\nu}=0$ and hence the uncertainty $\delta \alpha$ is reduced by the factor $1 / \sqrt{1-r^{2}} \simeq 2.3$. Similarly, if the value of $\alpha$ were accurately and independently known, and we fit for $\mu_{\nu}^{2}$ only, an identical improvement in the upper limit of $\left|\mu_{e}^{\text {sol }}\right|^{2}$ would result.

Conclusions. - In this paper, we present three new results. First, while neutrino magnetic moments are most

TABLE I. Limits on the magnetic moment (at 90\% C.L., in units of $10^{-10} \mu_{B}$ ) and statistical errors $(1 \sigma)$ on $\alpha$ for various data sets [5]: $504 \mathrm{~d}$ (I); the same just up to $13.0 \mathrm{MeV}$, to exclude the bins with an apparent excess compared to the constant data/SSM ratio (II); $708 \mathrm{~d}$; and $825 \mathrm{~d}$. In all cases we ignore the bin from 14.0-20.0 MeV.

\begin{tabular}{lccc}
\hline \hline Case & $\left|\mu_{e}^{\text {sol }}\right|^{2}$ & $\left|\mu_{e}^{\text {sol }}\right|$ & $\delta \alpha$ \\
\hline $504 \mathrm{~d}$, I & $\leq 3.9$ & $\leq 2.0$ & 0.025 \\
$504 \mathrm{~d}$, II & $\leq 4.2$ & $\leq 2.0$ & 0.027 \\
$708 \mathrm{~d}$ & $\leq 2.5$ & $\leq 1.6$ & 0.018 \\
$825 \mathrm{~d}$ & $\leq 2.3$ & $\leq 1.5$ & 0.017 \\
\hline \hline
\end{tabular}

fundamentally defined for mass eigenstates, in several cases of practical interest nonoscillating (i.e., independent of distance) effective magnetic moments can be defined for the flavor eigenstates. For Dirac neutrinos with only diagonal moments, these results are Eq. (4) for vacuum mixing and Eq. (9) for MSW mixing. For Majorana neutrinos with two flavors, the result is $\mu_{e}^{2}=\left|\mu_{12}\right|^{2}$, for either vacuum or MSW mixing. Second, MSW mixing can change the definition of the effective magnetic moment (allowing a dependence on the neutrino energy), so that the measured moments using $\bar{\nu}_{e}$ from a reactor and $\nu_{e}$ from the Sun could be different. Third, the shape of the SK recoil electron spectrum can be used to place a limit on the neutrino magnetic moment (note that we do not invoke any mechanism for neutrino interaction with the solar magnetic field). In general, this is a new limit, independent of the limit from reactor studies (with the same meaning only for solar vacuum oscillations). In any case, the limit obtained using the preliminary $825-\mathrm{d}$ data, $\left|\mu_{e}^{\text {sol }}\right| \leq 1.5 \times 10^{-10} \mu_{B}$, is comparable to the existing reactor limit of $1.8 \times 10^{-10} \mu_{B}$ [12].

This work was supported in part by the U.S. Department of Energy under Grant No. DE-FG03-88ER40397. J. F. B. was supported by Caltech. We thank Boris Kayser, Bob Svoboda, and Mark Vagins for discussions, and the Super-Kamiokande Collaboration for supplying the 825-d results.

[1] B. Kayser, Phys. Rev. D 26, 1662 (1982).

[2] R. E. Shrock, Nucl. Phys. B206, 359 (1982).

[3] A. V. Kyuldjiev, Nucl. Phys. B243, 387 (1984).

[4] P. Vogel and J. Engel, Phys. Rev. D 39, 3378 (1989).

[5] (504 d) Y. Fukuda et al., Phys. Rev. Lett. 82, 2430 (1999); (708 d) M. B. Smy, hep-ex/9903034; (825 d) M. Nakahata, in "Proceedings of TAUP99" (unpublished).

[6] W. Grimus and P. Stockinger, Phys. Rev. D 57, 1762 (1998).

[7] R. Barbieri and G. Fiorentini, Nucl. Phys. B304, 909 (1988); Ref. [4]; S. Pastor, J. Segura, V. B. Semikoz, and J. W. Valle, Phys. Rev. D 59, 013004 (1999).

[8] C. Caso et al., Eur. Phys. J. C 3, 1 (1998).

[9] S. N. Gninenko, Phys. Lett. B 452, 414 (1999).

[10] A. B. Balantekin and J. F. Beacom, Phys. Rev. D 54, 6323 (1996); A. B. Balantekin, J. F. Beacom, and J. M. Fetter, Phys. Lett. B 427, 317 (1998).

[11] J. N. Bahcall, P. I. Krastev, and A. Y. Smirnov, Phys. Rev. D 58, 096016 (1998).

[12] A. I. Derbin et al., JETP Lett. 57, 768 (1993) [Pis'ma Zh. Eksp. Teor. Fiz. 57, 755 (1993)].

[13] G. G. Raffelt, Stars as Laboratories for Fundamental Physics (University of Chicago Press, Chicago, 1996).

[14] J. Pulido and A. M. Mourao, Phys. Rev. D 57, 1794 (1998), and references therein.

[15] J. N. Bahcall et al., Phys. Rev. C 54, 411 (1996).

[16] M. Nakahata et al., Nucl. Instrum. Methods Phys. Res., Sect. A 421, 113 (1999). 